\title{
Sudden Bispectral Index Reduction and Suppression Ratio Increase Associated with Bradycardia in a Patient Undergoing Breast Conserving Surgery
}

\author{
Youngheun Jo, MSc ${ }^{1,2}$, Jae-Man Kim, BSc ${ }^{1}$, Sang-Beom Jeon, MD², Se-Ung Park, MD ${ }^{1}$, Hye-Jin Kam, PhD ${ }^{3}$, Woo-Hyun Shim, PhD ${ }^{3}$, \\ Sung-Hoon Kim, MD ${ }^{1,3}$ \\ Departments of ${ }^{1}$ Anesthesiology and Pain Medicine, ${ }^{2}$ Neurology, Asan Medical Center, University of Ulsan College of Medicine, Seoul; ${ }^{3}$ Health Innovation Bigdata Center, \\ Asan Institute for Lifesciences, Asan Medical Center, Seoul, Korea
}

Background: The bispectral index (BIS) is a valuable indicator for measuring sedation levels and patient consciousness. Recent reports have highlighted its clinical value as an indicator for anesthesia-related cerebral hypoperfusion and ischemic brain damage.

Case Report: A 55-year-old female patient underwent right breast conservation surgery during general anesthesia. During surgery, the patient experienced abrupt bradycardia (heart rate of 36 bpm) without hypotension. During bradycardia, her BIS was severely reduced from 45 to 20 along with elvated suppression ratio (50). After injection of $0.5 \mathrm{mg}$ of atropine, her BIS level was recovered, her heart rate was increased, and her suppression ratio was decreased.

Conclusion: The patient recovered from anesthesia without showing any signs of neurological sequelae based on BIS level monitoring.

J Neurocrit Care 2018;11(1):39-42

Key words: Consciousness monitors; Bradycardia; Hypotension; Atropine

\author{
Received February 27, 2018 \\ Revised May 3, 2018 \\ Accepted May 15, 2018 \\ Corresponding Author: \\ Sung-Hoon Kim, MD \\ Department of Anesthesiology and \\ Pain Medicine, Asan Medical Center, \\ University of Ulsan College of Medicine, \\ 88 Olympic-ro 43-gil, Songpa-gu, Seoul \\ 05505, Korea \\ Tel: $+82-2-3010-3863$ \\ Fax: $+82-2-3010-6790$ \\ E-mail: shkimans@amc.seoul.kr
}

Copyright $\odot 2018$ The Korean Neurocritical Care Society

\section{INTRODUCTION}

The bispectral index (BIS) is known to be a measure of overall level of sedation and consciousness. ${ }^{1,2}$ It is also an indicator for anesthesia-related cerebral hypoperfusion. Moreover, BIS is a useful indicator of ischemic brain damage when it is accompanied by electroencephalogram (EEG). In this case report, our patient experienced reduced BIS correlated with elevated suppression ratio (SR) during abrupt bradycardia. This suggests that heart rate (HR) and BIS levels might be correlated in patients undergoing breast conservatory surgery during anesthesia. Written informed consent has been obtained from the patient.

\section{CASE REPORT}

A 55-year-old female patient (body weight, $52 \mathrm{~kg}$; height, $157 \mathrm{~cm}$ ) underwent breast conservatory surgery due to ductal carcinoma of the right breast. The patient did

cc This is an Open Access article distributed under the terms of the Creative Commons Attribution Non-Commercial License (http://creativecommons.org/licenses/bync/4.0) which permits unrestricted non-commercial use, distribution, and reproduction in any medium, provided the original work is properly cited. 
not suffer any co-morbidity. Her blood pressure levels were $137 / 87 \mathrm{mmHg}$ and her HR was 65 bpm. During operation, her blood pressure, electrocardiogram, pulse oximetry, and end-tidal carbon dioxide were regularly monitored non-invasively. BIS sensor was attached to her forehead to monitor the depth of anesthesia during operation. After pre-oxygenation, general anesthesia was induced with midazolam (2 mg), 1\% propofol ( $50 \mathrm{~mL}$ at $2.5-3 \mathrm{mg} / \mathrm{mL}$ ), and remifentanil ( $2 \mathrm{mg}$ at $10-15 \mathrm{ng} / \mathrm{mL}$ target concentration infusion) using infusion pump. Tracheal intubation was facilitated with rocuronium (25 mg). During surgery, her blood pressure and HR were maintained at 120 140/50-60 mmHg and 50-60 bpm, respectively. After 1 hour since starting the surgery, reduced HR (30-40 bpm) was accompanied by a decrease in BIS (from 40 to 25) and an increase in SR (from 0 to 40-45). After administration of atropine at $0.5 \mathrm{mg}$, we observed an increase in HR (40 to 70 bpm) and normalization of BIS and SR levels within less than 10 minutes. After completion of surgery, glucopyrrolate $(0.4 \mathrm{mg})$ and neostigmine $(1.5 \mathrm{mg})$ were administered for neuromuscular reverse. The patient did not experience any neurological sequelae after the surgery. No ischemic damage was found in the patient's postoperative magnetic resonance images. Data were plotted using Python 3.6.0 (Python Software Foundation, https://www.python.org/). Minimal processing was performed prior to analysis.

\section{DISCUSSION}

BIS monitor was developed to evaluate the appropriateness of surgical environment by assessing neurophysiological response to noxious stimuli. BIS score associated with an appropriate level of sedation was set at 60-80 and moderate hypnosis for surgery was set at 40-60. Our patient showed reduced BIS accompanied by severe bradycardia. Her BIS levels recovered following an increase in HR after administration of atropine (Fig. 1). Reduction in BIS level is known to suggest deepened sedation. Previous reports have suggested that cerebral hypoperfusion decreases BIS. Mérat et al. ${ }^{1}$ have reported that monitoring
BIS may facilitate the detection of severe cerebral ischemia. They observed two cases in which BIS levels were decreased in parallel with somatosensory evoked potential during carotid clamping in patients during carotid endarterectomy. Hayashida et al. ${ }^{4}$ have examined cerebral hypoperfusion using BIS levels during pediatric cardiac surgery. In five children, they reported 14 cases of simultaneous decrease in regional cerebral hemoglobin oxygen saturation $\left(\mathrm{rSO}_{2}\right)$ and BIS levels during acute hypotension. Hemmerling et al. ${ }^{5}$ have also described cerebral hypoperfusion causing abrupt decreases in BIS from 45-50 to 0 during distal grafting.

Hashimoto et al. ${ }^{6}$ have also reported a marked reduction in BIS accompanied by severe bradycardia without hypotension. They described that a diabetic patient undergoing ophthalmic surgery showed sudden severe bradycardia without hypotension followed by drastic decrease in BIS and increase in SR. With isoproterenol infusion, the patient

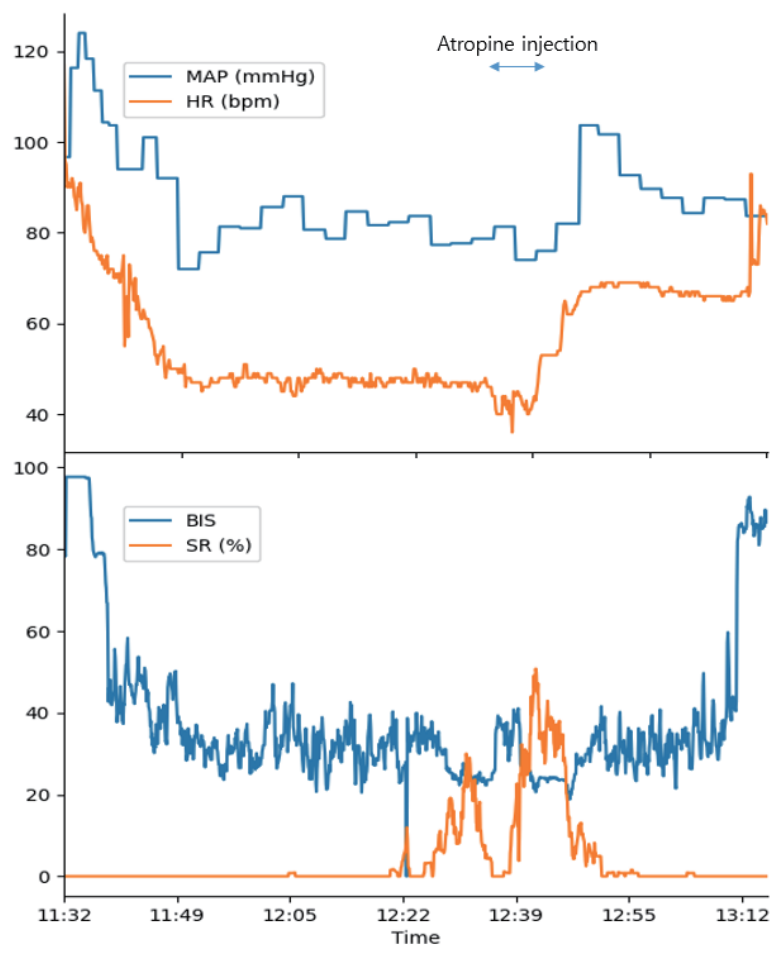

Figure 1. A sudden decrease in bispectral index (BIS) and increase in suppression ratio (SR) accompanied by severe bradycardia ( 35 beats per minute). After atropine infusion (doubleheaded blue arrow), BIS and heart rate (HR) levels recovered to levels prior to surgery. MAP, mean arterial pressure. 
recovered prior states of BIS and HR without any neurological sequelae post-surgery.

In our report, the result suggests that monitoring BIS and SR levels may aid in detecting bradycardia during surgery. SR is one of the sub-parameters incorporated in the BIS, which quantifies the percentage of suppression during burst suppression. The monitoring of both parameters derived from EEG may improve our insight into the patient's consciousness.

During surgery, implanting a pacemaker can also improve mental performance, ${ }^{7}$ EEG findings, ${ }^{8}$ and cerebral blood flow $(\mathrm{CBF})^{9}$ in patients with decreased CBF due to bradycardia. However, there have been contradicting reports with different anesthetics ${ }^{10,11}$ due to device's electric interference ${ }^{12}$ and patient's conditions in which BIS values do not coincide with clinically judged sedative-hypnotic state. Therefore, clinicians must be aware of this and further investigate the relationship between BIS levels and bradycardia. While normalization of the HR is vital for cerebral functioning, Koide et al..$^{13}$ have suggested that optimal range of HRs might be needed to minimize vascular resistance and maximize blood flow. They have found a significant correlation between changes in HR and cerebral vascular resistance or $\mathrm{CBF}$, but not cardiac output, in patients experiencing severe bradycardia during pacemaker implantation. These patients were also found to show improved cognitive function post implantation. In our case, cerebral activity might have been reduced in response to decreased CBF as BIS was decreased from 40 to 25 after bradycardia. It was increased after atropine injection.

Our case of severe bradycardia during anesthesia is uncommon. Particularly, the drop in BIS and peak in SR levels followed by short recovery to previous levels with BIS dropping and SR peaking again are not well defined. During our patient's surgery, BIS levels maintained at around 35 to 45. They dropped to less than 25 before atropine injection. Although less drastic, atropine infusion caused an increase in BIS level, similar to report in a previous study. ${ }^{6}$ Severe bradycardia during anesthesia may cause cerebral hypoperfusion which may result in cerebral impairments. Therefore, monitoring both BIS and SR levels in surgery may aid in the detection of bradycardia-induced cerebral hypoperfusion.

\section{REFERENCES}

1. Mérat S, Lévecque JP, Le Gulluche Y, Diraison Y, Brinquin L, Hoffmann JJ. BIS monitoring may allow the detection of severe cerebral ischemia. Can J Anaesth 2001;48:1066-9.

2. Rampil IJ. A primer for EEG signal processing in anesthesia. Anesthesiology 1998;89:980-1002.

3. Myles PS, Daly D, Silvers A, Cairo S. Prediction of neurological outcome using bispectral index monitoring in patients with severe Ischemic-Hypoxic brain injury undergoing emergency surgery. Anesthesiology 2009;110:1106-15.

4. Hayashida M, Chinzei M, Komatsu K, Yamamoto H, Tamai H, Orii R, et al. Detection of cerebral hypoperfusion with bispectral index during paediatric cardiac surgery. Br I Anaesth 2003;90:694-8.

5. Hemmerling TM, Olivier JF, Basile F, Le N, Prieto I. Bispectral index as an indicator of cerebral hypoperfusion during off-pump coronary artery bypass grafting. Anesth Analg 2005; 100:354-6.

6. Hashimoto H, Nakamura H, Hirota K. Marked reduction in bispectral index with severe bradycardia without hypotension in a diabetic patient undergoing ophthalmic surgery. / Anesth 2008;22:300-3.

7. Lagergren K. Effect of exogenous changes in heart rate upon mental performance in patients treated with artificial pacemakers for complete heart block. Br Heart / 1974;36:112632.

8. Isaksson A, Lagergren K, Wennberg A. Visible and non-visible EEG changes demonstrated by spectral parameter analysis. Electroencephalogr Clin Neurophysiol 1976;41:225-36.

9. Shapiro W, Chawla NP. Observations on the regulation of cerebral blood flow in complete heart block. Circulation 1969;40:863-70.

10. Hering W, Geisslinger G, Kamp HD, Dinkel M, Tschaikowsky K, Rügheimer E, et al. Changes in the EEG power spectrum after midazolam anaesthesia combined with racemic or S- (+) ketamine. Acta Anaesthesio/ Scand 1994;38:719-23.

11. Rampil IJ, Kim JS, Lenhardt R, Negishi C, Sessler DI. Bispectral EEG index during nitrous oxide administration. Anesthesioogy 1998;89:671-7.

12. Hemmerling TM, Migneault B. Falsely increased bispectral 
index during endoscopic shoulder surgery attributed to interferences with the endoscopic shaver device. Anesth Analg 2002;95:1678-9, table of contents.

13. Koide H, Kobayashi S, Kitani M, Tsunematsu T, Nakazawa Y.
Improvement of cerebral blood flow and cognitive function following pacemaker implantation in patients with bradycardia. Gerontology 1994;40:279-85. 